\title{
De la espaciación del tiempo a la temporalización del espacio. Espacio y memoria en la obra de Monika Maron'
}

\author{
Leopoldo Domínguez \\ Universidad de Sevilla
}

http:// dx.doi.org/ 10.12795/mAG Azin.2015.i23.03

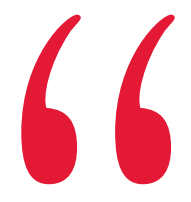

Identität ist, wie leicht einzusehen, eine Sache von Gedächtnis und Erinnerung", postula Jan Assmann (1992: 59). La memoria, entendida como la capacidad que posee el sujeto para reconstruir el pasado, se convierte en un elemento indispensable en el proceso de constitución de su identidad. Según Assmann, la memoria depende de unos marcos sociales en cuyo contexto registra sus experiencias, así como de las necesidades de significado del presente desde donde el sujeto rememora. La identidad individual surge, pues, de las historias sucesivas que dicho sujeto compone sobre sí mismo. La importancia que desempeña la narración en este proceso lleva a Ricoeur a darle el nombre de identidad narrativa (Forte 2005: 45). Entre las principales funciones de la literatura desde sus orígenes se encuentra la reconstrucción del pasado (Basseler / Birke 2005: 123). "Die Erinnerungen”, señala Humphrey, "sind der Stoff, aus dem die Literatur ist" (Humphrey 2005: 73). La literatura se presta como medio para plasmar los hechos pretéritos y como escenario para representar los procesos de recuerdo. Esta representación del recuerdo se consigue cuando la obra literaria presenta dos elementos. Por un lado, la obra cuenta con un centro de percepción subjetiva. Por otro lado, en ella la narración posee al menos dos planos temporales: el pasado rememorado y el presente desde el cual se recuerda.

Para la representación de los procesos de recuerdo resulta imprescindible el elemento espacial. El espacio actúa como bisagra entre los diferentes planos temporales. Bajtin expresa en su concepto de "cronotopo" la relación recíproca que mantienen en la obra literaria el espacio y el tiempo. Esta relación equivale a lo que Basseler y Birke (2005: 131) denominan como la "espaciación del tiempo" (Verräumlichung der Zeit) y la "temporalización del espacio" (Verzeitlichung des Raums). El tiempo y el espacio están estrechamente relacionados en la representación de los procesos de recuerdo. Por un lado, la espaciación del tiempo se distingue en la obra literaria cuando se describe una cicatriz corporal o se utiliza la habitación como espacio de recuerdo. En ambos casos, se tiene acceso al pasado en la obra literaria de forma estática y permanente. Por otro lado, la temporalización del espacio acontece, por ejemplo, cuando un narrador regresa a su lugar de origen. Su desplazamiento por el espacio, en el encuentro con el pretérito, implica esta vez un desplazamiento simultáneo a través del tiempo.

La conversión del espacio en tiempo durante la temporalización del espacio es posible por la capacidad del espacio para almacenar el tiempo. "Nicht nur daß sie [Räume] die Erinnerung festigen und beglaubigen, indem sie sie lokal im Boden verankern, sie verkörpern auch eine Kontinuität der Dauer, die die vergleichsweise kurzphasige Erinnerung von Individuen, Epochen und auch Kulturen, die in Artefakten konkretisiert ist, übersteigt" (A. Assmann 1999: 299). La continuidad del tiempo, que posibilita el espacio, lo convierte en un marco relativamente estable, que contribuye a que el sujeto pueda preservar sus recuerdos e ir conformando su identidad. Del mismo modo, el espacio está expuesto a los cambios históricos, sociales y culturales, que actúan en detrimento de la cualidad material y acentúan su naturaleza subjetiva. "So gegenständlich konkret er ist, so vielfältig präsentiert er sich in den unterschiedlichen Perspektivierungen" (A. Assmann 1999: 330). Por ello por lo que Assmann compara el espacio (especialmente el urbano) con un palimpsesto, y su lectura con la de los distintos 


\section{Resumen:}

En los escritos de Monika Maron la línea entre la ficción y la realidad es a menudo tan difusa como la que separa la historia personal de la historia nacional. En el continuo proceso de reconstrucción del yo y de valoración de los acontecimientos históricos, que Maron escenifica en sus obras, es llamativo el papel que desempeña en ellas el espacio. La función del espacio, que le permite representar el proceso de recuerdo, cambia significativamente desde las primeras novelas de la autora berlinesa hasta sus últimos escritos literarios. El objeto de esta contribución es el análisis del espacio y la memoria en la obra en prosa de Maron desde Flugasche (1981) hasta Geburtsort Berlin (2003) y Zwischenspiel (2013).

Palabras clave: Monika Maron, literatura, historia, memoria, espacio, Berlín, Alemania, RDA, reunificación.

\section{Zusammenfassung:}

In den Schriften Monika Marons ist oft die Linie zwischen Fiktion und Realität so diffus wie die Grenze zwischen der persönlichen und der nationalen Geschichte. Bei dem kontinuierlichen Prozess der Ich-(Wieder-)Konstruktion und der Bewertung von historischen Ereignissen, die Maron in ihren Werken inszeniert, ist die spielende Rolle des Raumes besonders auffallend. Die Funktion des Raumes, der es ihr ermöglicht, den Erinnerungsprozess darzustellen, unterscheidet sich wesentlich von den ersten Romanen der Ostberliner Autorin zu ihren letzten literarischen Werken. In diesem Beitrag wird auf die Analyse von Raum und Gedächtnis im Marons Prosawerk seit Flugasche (1981) bis Geburtsort Berlin (2003) und Zwischenspiel (2013) eingegangen.

Schlüsselwörter: Monika Maron, Literatur, Geschichte, Gedächtnis, Raum, Berlin, Deutschland, DDR, Wiedervereiningung. trazos en un texto. Su lectura no solo implica la presencia de un sujeto, capaz de descifrarlo, sino que depende de los distintos afectos que es capaz de concentrar dicho espacio y que depende de lo que el sujeto busca, conoce o le relaciona con el mismo.

La subjetividad del espacio se incrementa en el caso de lo que Aleida Assmann denomina, a partir del concepto de Nora, como "lugares de memoria". Apoyándose en la idea de "aura" de Walter Benjamin, Assmann destaca el poder que comparten estos lugares, que se convierten en zonas de contacto. "Die eigentümliche Verbindung von Nähe und Ferne, macht diese zu auratischen Orten, an denen man einen unmittelbaren Kontakt mit der Vergangenheit sucht" (A. Assmann 1999: 337-338). Según Assmann, dichos lugares permiten aglutinar el espacio y el tiempo para hacer confluir presencia y ausencia, el presente sensorial y el pasado histórico.

\section{Espaciación del tiempo: la habitación como espacio de recuerdo}

Tanto la memoria como los procesos de recuerdo están presentes en el conjunto de la obra de Monika Maron. Sus novelas tratan normalmente de protagonistas femeninas que rememoran (Boll 2002: 15). Las figuras recuerdan y reflexionan en el transcurso de las mismas sobre los acontecimientos históricos. Dichas figuras tratan de valorar en retrospectiva los hechos pasados y de revisar, a través de los acontecimientos, la propia biografía. "Tan unida se encuentra su biografía a la historia reciente de Alemania que se ha convertido en el tema fundamental de su producción literaria”, sostiene Sainz (2009: 220). Según la autora, sus circunstancias vitales llevan a Maron a convertirse en un testigo de excepción de la posguerra, la constitución de los dos Estados alemanes y las entretelas de la vida política de la RDA hasta su desaparición con la caída del Muro y la consiguiente reunificación. Cada hito histórico, que se sucede en la trayectoria vital de la escritora, propicia el análisis del presente y, junto con ello, la relectura del pasado, entretejido siempre con el propio devenir del país.

En Flugasche [Cenizas volantes] (1981) su protagonista Josefa reprime la realidad al anteponer su creencia ciega en la utopía socialista. Sin embargo, en "Die Überläuferin" [La tránsfuga] (1986), Rosalind se vale de una parálisis sobrevenida en las piernas para recluirse entre los muros de su habitación y reconstruir, dentro del espacio cerrado, el yo reprimido de Josefa. "Nachdem Rosalind von der Haltbarkeit ihres Zustandes überzeugt war, begann sie darüber nachzudenken, wie die unvorstellbare Menge Zeit, die ihr plötzlich zur Verfügung stand, zu verwerten sei” (Maron 1986: 12). Rosalind contempla el tiempo como 
algo calculado y compara el pasado con una biblioteca (cfr. Basseler / Birke 2005: 133), donde reúne sus vivencias como si fueran libros. Este espacio, al que la figura dice poder acceder en cualquier momento, se convierte en la segunda novela de Maron en un espacio de memoria (Boll 2002: 40).

Tras la reciente caída del Muro de Berlín, en Stille Zeile Sechs [Stille Zeile, número seis] (1992) la misma Rosalind considera que ha llegado el instante de poner punto y final a su aislamiento para confrontarse más activamente con el recuerdo de la RDA. El espacio donde se desarrolla la novela es la vivienda de Herbert Beerenbaum. Este antiguo funcionario de la RDA realiza el encargo a la historiadora de la escritura de sus memorias. Como Rosalind afirma en

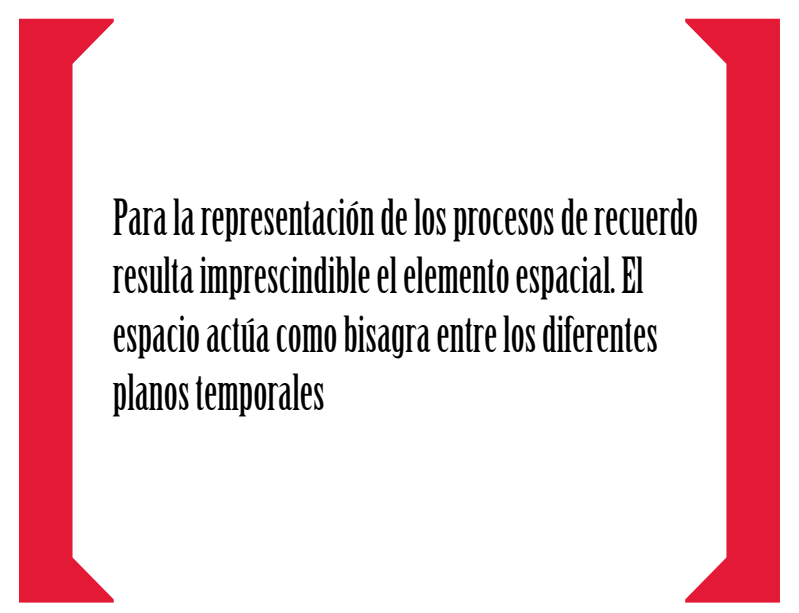

la novela, en la vivienda todo le es familiar, ya que se parece a la de sus padres (Maron 1992: 45-46.). La autora mantiene a lo largo de su vida una tensa relación con su padrastro, Karl Maron, que llega a ocupar durante la RDA el puesto de ministro del Interior. Maron personifica en este alter ego de Karl su rechazo del sistema político represivo del Partido Socialista Unificado. El fallecimiento al final de Beerenbaum, del cual Rosalind llega incluso a sentirse responsable, puede entenderse como una total liberación de Maron respecto de dicho periodo. La muerte y el entierro del anciano significan el ocaso de un capítulo de la historia al que Maron pretende dar por superado. A su vez, la incertidumbre sobre el futuro del país permite proyectar nuevas ilusiones de cambio, para el cual tanto la generación de Maron como la de la protagonista de la novela llevan esperando desde hace tanto (Sainz 2009: 224).

En Animal triste (1996) es otra vez la caída del Muro el acontecimiento que actúa como incentivo del acto de escritura y de revisión de la trayectoria vital de la figura en la obra. Como Maron señala en una entrevista a propósito de esta creación literaria:

Der Fall der Mauer war überhaupt die Voraussetzung dieser Explosion oder Implosion. Die Zeiten haben sich verändert, und zwar auf eine grandiose Art, und diese Veränderung hat eine Leidenschaft und Leidenschaftlichkeit dieser Frau freigesetzt. Die Freiheit war die Voraussetzung des Glücks. Der Bruch in der Biographie hat überhaupt erst befähigt, diese Lebendigkeit wieder in sich selbst zu finden. (Eschering 1996: 10)

De nuevo encerrada entre muros y desde un estado atemporal, la protagonista de Animal triste trata de ordenar y valorar su confusión de recuerdos y pensamientos. Apoyándose en algunos objetos personales que conserva de una relación amorosa, hace balance de los años desde la reunificación. "Manchmal glaube ich sogar, daß auch die Mauer in Berlin nur eingerissen wurde, damit Franz mich endlich finden konnte" (Maron 1996: 51). La caída del Muro permite el encuentro entre la protagonista y Franz, que además de estar casado procede de la Alemania occidental. Los dos se conocen en el Museo de Ciencias Naturales de la ciudad, donde la figura trabaja como paleontóloga. La narradora participa en la reconstrucción del esqueleto de un braquiosaurio. La belleza del animal, que es comparable a la nueva realidad emergente, cautiva por igual a los amantes y les une en una relación intensa. Pero progresivamente la pasión se va envenenando debido a los celos de la protagonista, que no es capaz de soportar la doble vida de Franz (Sainz 2009: 224).

Maron reflexiona a través de esta relación frustrada sobre el nuevo espacio nacional compartido. Si por un lado, este espacio permite el encuentro entre los amantes, por otro lado, se descubre todavía como una utopía inalcanzable. El museo, que está ubicado en Berlín Este, cerca de la Puerta de Brandeburgo donde comienza a demolerse el muro, se convierte en símbolo de la aproximación y la distancia entre las dos Alemanias. La novela permite establecer una analogía con Der geteilte Himmel [El cielo partido] (1963) de Christa Wolf. En la obra de Wolf el espacio se interpone en el proyecto vital común de Manfred y Rita. Mientras Manfred abandona la RDA, Rita decide quedarse para participar en la construcción del estado socialista. En Animal triste la desilusión amorosa del desencuentro echa por tierra las falsas expectativas creadas, lo que lleva a la protagonista a refugiarse en el lugar donde surge el romance. Asimismo, como en Die Überläuferin, el espacio desde 
donde se construye la narración funciona como elemento tanto creador como destructor de memoria (Rossbacher 1997: 206); y en dicho espacio de rememoración los procesos de recuerdo y de olvido resultan aún actos voluntarios.

Ich weiß gar nicht, ob wir uns so falsch erinnern, aber die Willkür unseres Erinnerns und auch unseres Vergessens ist ja doch verblüffend. Ich habe das bei meiner Mutter erlebt, die jetzt Schriftstücke aus ihrer Jugend gefunden hat. Sie sollte aus Deutschland ausgewiesen werden, weil sie Polin und Halbjüdin war, und sie wußte das nicht mehr. (Eschering 1996: 10)

El título de la novela Endmoränen [Morrenas terminales] (2002) es ya en sí mismo una metáfora de la memoria y del proceso de recuerdo, que Maron escenifica. Según Merck, "hay que entender el título más desde un punto de vista antropológico que geológico, ya que la autora pretende comparar su momento personal con esa formación geológica. Se trata de un punto de inflexión personal, de reconstrucción y revisión de los propios valores que sustentan su vida" (Merck 2009: 383-384). El pretexto de la escritura por encargo de una editorial de una biografía histórica lleva a Johanna a escapar de la monótona vida conyugal para recluirse en su casa de verano de Basekow, en la frontera con Polonia. Durante la estancia en la casa retoma una relación epistolar con un antiguo amigo del matrimonio. Como el personaje de Franz de Animal triste, Christian es alemán occidental. Johanna le cuenta en las cartas la frustración que vive en la Alemania tras la caída del Muro. La figura critica que el capitalismo y el consumo representan los únicos valores de la nueva sociedad. Además, le confiesa haber perdido la motivación que poseía en la RDA, la cual se sustentaba en un afán de resistencia incansable contra el sistema. El encuentro de Johanna con el destinatario de las cartas tampoco llega a consumarse en esta obra. Sin embargo, Christian le aconseja algo que le ayuda a reorientarse en el espacio dejado.

Fahr wieder nach Berlin, geh auf die Friedrichsbrücke oder über die Linden und den Gendarmenmarkt. Trink bei Luther und Wegener (allein wegen des Namens) einen Kaffee oder einen Wein und denke da über deine Gräfin nach. Vielleicht war deine Verbannung ja gut, um die anonyme Gesellschaft der Stadt wieder genießen zu können, ganz leidenschaftslos genießen, Johanna, fahr nach Hause und schreib mir, ob Du wieder verjüngst. (Maron 2002: 222)
Según Sainz, la protagonista regresa a Berlín transformada. Pues aunque sabe que le espera en la ciudad una vida con los mismos elementos de su vida anterior, parece dispuesta a intentar en ella un nuevo comienzo (Sainz 2009: 229).

\section{Temporalización del espacio: la lectura del pasado a través de la ciudad}

Berlín resulta una pieza fundamental en la obra de Maron. La arquitectura de la capital, que permite reflejar como ninguna otra ciudad alemana la historia reciente del país, suele ser el escenario directo o indirecto de sus obras. La vinculación de Maron con Berlín se remonta hasta su propio nacimiento. La autora nace en 1941 en el barrio de Neukölln (Berlín Oeste). En 1951 su madre, Hella Iglarz, tras estar prometida con un soldado del frente con el que no puede casarse debido a las leyes raciales (dado el origen judío del abuelo de Maron), se muda con ella a la parte oriental de la ciudad.

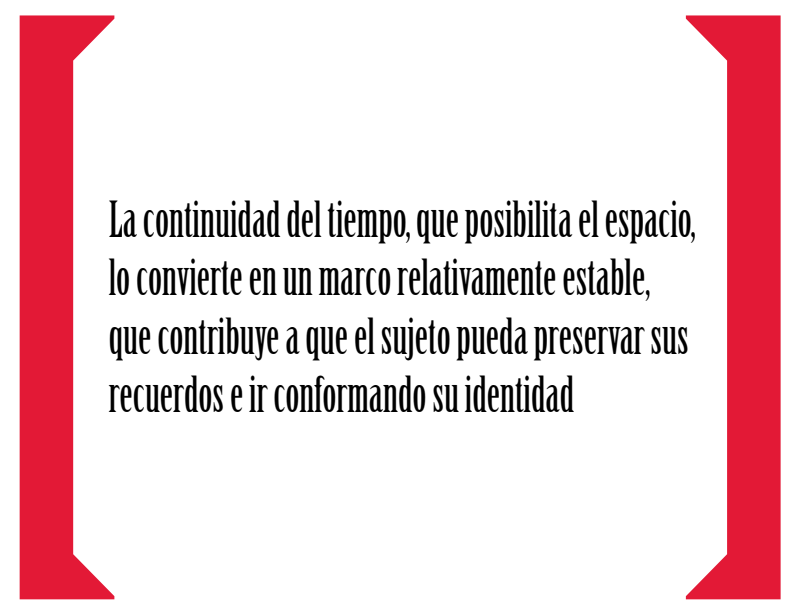

En el ensayo Geburtsort Berlin [Lugar de nacimiento, Berlín] (2003) Maron recorre este espacio urbano para recuperar su infancia y reflexionar nuevamente sobre el pasado y el futuro de Alemania. Según Pytel-Bartnik, el espacio no solo funciona en la obra como inhibidor del recuerdo sino además como nexo de unión del discurso narrativo:

Die Autorin erzählt ihre Geschichte nicht in einer chronologischen Abfolge von Lebensstationen, sondern eher in einem Free-Flow von den erinnerungswürdigen, ihr Leben im östlichen und nach der Wende im westlichen Teil Berlins prägenden Ereignissen, Lebensetappen, Situationen, manchmal Stimmungsbildern. (Pytel-Bartnik 2010: 184) 
Maron se dedica desde 1976, justo al año siguiente de la muerte de su padrastro, exclusivamente a la escritura. La autora goza al principio de ciertos privilegios en la RDA, como diversos permisos para viajar al extranjero . Pero su aislamiento cada vez mayor y las dificultades para publicar sus obras la fuerzan a marcharse en 1988 a Hamburgo. Allí permanece hasta incluso después de la caída del Muro, concretamente hasta el año 1992, cuando Maron decide regresar de nuevo a la ciudad que añora.

Maron describe en la obra cómo frente al vacío interior que le provoca la fastuosa ciudad de Hamburgo, Berlín se le revela como un archivo abundante de recuerdos: "In Berlin könnte ich mich, wenn ich es darauf hinlegte, hundertmal am Tag treffen, in jedem Alter, glücklich oder heulend, allein, in Gesellschaft, verliebt, verlassen, überall hocke ich und warte darauf, daß ich vorbeikomme" (Maron 2003: 55). Maron comenta al mismo tiempo algunos de los cambios que se producen sobre el entramado urbano desde la segunda mitad del siglo anterior.

\begin{abstract}
Also begebe ich mich an einem Mittwoch im September, eine Stunde vor Beginn des Spektakels, an den beschriebenen Ort. [...] Auf der Promenade zwischen den beiden Fahrbahnen reitet in philosophisch nachdenklicher Pose Eff Zwo. Kurz nach dem Krieg hatte man das Denkmal von Christian Daniel Rauch als Sinnbild militarischer Preußenschaft nach Potsdam verbannt und vor sechs Jahren, nachdem die ältere deutsche Geschichte weniger emotional betrachtet, dafür auf ihre praktische Verwendbarkeit untersucht wurde, hierher, auf seinen seit 1851 angestammten Platz, zurückgebracht. (Maron 2003: 52)
\end{abstract}

Sus comentarios descubren a la vez la ciudad como un palimpsesto donde se puede leer el paso de su historia. La lectura de los trazos en el texto de Berlín, por el que se pasea Maron, aparece influida por las diferentes vivencias del pretérito. Un ejemplo es la estación Friedrichstraße. Como describe la autora, este antiguo paso fronterizo entre Berlín Este y Oeste continúa presente, a pesar de su desaparición, en el plano mental de buena parte de los habitantes orientales:

Halt! Weitergehen verboten; ich weiß es, aber ich sehe es nicht, ich sehe nur auf die Tür, wie die Blicke aller Umstehenden magisch auf die Tür gerichtet sind. [...] Die meisten sind Rentner, Ostmenschen also. Sie schleppen schwere Taschen und Beutel, wenn sie vom Einkaufen am Zoo oder in der Neuköllner Karl-Marx-
Straße kommen, große Koffer, wenn sie Verwandte in Westdeutschland besucht haben. Einmal habe ich hier einen Gepäckträger gesehen ebenso alt und auch so gebrechlich wie die Frau, deren Koffer er trug. Haben die schwer Beladenen sich samt ihrem Gepäck endlich durch den schmalen Spalt in der Tür bugsiert, retten sie sich zunächst an das eiserne Geländer, das ein zimmergroßes Areal vor dem Tor begrenzt. Da stellen sie ihr Gepäck ab, sortieren die Papiere, die sie in den nervösen, schweißfeuchten Händen halten, suchen unter den Wartenden jenseits der Absperrung nach einem, der ihretwegen da steht [...] (Maron 2003: 18-19)

Ese mismo Berlín que reverbera en la memoria de Maron contrasta con la imagen que tiene de la antigua zona occidental. Maron reside desde su retorno de Hamburgo en el barrio de Schöneberg (Berlín Oeste). No obstante, no consigue identificarse con una topografía de la ciudad que carece para la autora de toda carga emocional e identitaria.

Es ist ein später Nachmittag oder früher Abend im Herbst, die Schaufenster sind schon beleuchtet, am anderen Ende der Straße behauptet die Gedächtniskirche ihre symbolische Wichtigkeit. Wie ein schwarzer Wächter dominiert sie das Bild, und ich denke, daß die Straße, das Licht, die Kirche nicht für mich da sind, daß ich nicht reingehöre in dieses Bild, weil mein Visum am ersten Oktober abläuft. (Maron 2003: 69)

Desde la perspectiva del berlinés occidental, su nueva frontera invisible de la ciudad la sitúa Maron en el distrito del centro. "Die Stadtmitte ist ihr westlicher Außenbezirk. Die Mitte ist die Grenze; das Nichtüberschreitbare ist die Mitte, auch des Denkens. Da, mitten in die Mitte hinein führt eine Tür [...] Die Tür hat auf einer Seite eine Klinke, auf der anderen" (Maron 2003: 15).

Maron habla ya de esta división en el ensayo "Ich war ein antifaschistisches Kind" [Fui una niña antifascista] (1989). El texto es un preámbulo de su novela posterior Pawels Briefe [Las cartas de Pawel] (1999). En ella Maron narra el regreso a la casa natal. El espacio le sirve una vez más como incentivo del recuerdo para reconstruir la historia familiar. Maron medita en dicho ensayo sobre el reto que la división física plantea en la constitución de la nueva identidad alemana tras la caída del Muro. Maron admite la impotencia de los ciudadanos orientales, que, desde el alineamiento de la DDR al bloque soviético, se encuentran 
sumidos en la indefensión y el desprecio de sí mismos. A su vez, critica el desdén de los alemanes occidentales. Según Maron, estos no entienden que su bienestar económico no es solo una consecuencia de su trabajo sino sobre todo de un golpe de fortuna geográfica . "Läge Schwaben an der Oder, läge Leipzig am Rhein ... Dann hießen die Schlagzeilen in den Zeitungen heute vielleicht: Wieder hunderttausend Demonstranten in Stuttgart" (Maron 2003: 27). Esta división, que afecta en general al país, se refleja de igual forma en el caso de la capital germana.

A diferencia de las novelas y ensayos anteriores, Maron se muestra sin embargo por primera vez en Geburtsort Berlin optimista con respecto al proceso de reunificación. En este sentido Maron comenta en retrospectiva el episodio de la polémica que surge con el ocultamiento artístico del Reichstag. La iniciativa, ideada por Christo y JeanneClaude, tiene lugar entre el 24 de junio hasta el 9 de julio de 1995. En este periodo, en el cual el edificio parlamentario es recubierto con una lona, hay quienes intentan retirarla por el impulso curioso de contemplar su interior. Por un lado, la anécdota consigue actuar como un necesario bálsamo que causa un efecto terapéutico; por otro, permite a los berlineses reflexionar acerca de su identidad colectiva. La invisibilidad temporal de este espacio emblemático posibilita, gracias al ocultamiento, liberarlo del pesado lastre de su historia y permite que se proyecten sobre él nuevos significados (PytelBartnik: 2010: 188). Como sostiene Maldonado (2010: 177), la adaptación al nuevo contexto histórico-político precisa inevitablemente una transformación no tanto exterior sino sobre todo interior por parte de la sociedad alemana. La autora concluye el texto justamente con estas palabras: “[...] seit Christo den Reichstag verhüllt hat, ist Berlin eine andere Stadt. [...] Nichts, dachte ich, wird nach Christo sein wie vor" (Maron 2003: 78, 87).

Por otra parte, el predominio hasta ahora del espacio cerrado, concebido como un lugar protegido de retrospección, se rompe en Geburtsort Berlin. Mientras que en la representación del recuerdo, a través de la habitación, la coordenada espacial está a merced del tiempo, en el momento en que Maron sale al exterior es el elemento del espacio el que se impone sobre el otro. Dicho de otra forma, la espaciación del tiempo, que Maron escenifica mediante la habitación, da paso en el espacio abierto de la ciudad de Berlín a una situación de temporalización del espacio. Al verse superada por este nuevo escenario inédito expresa la autora: "Das Gesetz, nach dem ich mich erinnere, kann ich nicht erkennen” (Maron 2003: 75).
Maron siente en Geburtsort Berlin que su memoria no responde más al libre albedrío sino que resulta dependiente de los lugares físicos. Esto le fuerza a preservar, mediante la escritura, unos recuerdos del ayer que se debilitan por los sucesivos cambios en la arquitectura. La metamorfosis de la ciudad, provocada por las nuevas construcciones y acontecimientos, contribuye a la conformación de una nueva identidad. Aunque Maron valora el proceso de adaptación como necesario y positivo, no puede ocultar la nostalgia e incertidumbre que le crean. Por ello Maron se aferra a los vestigios de una memoria, que corre el riesgo de perderse al ser superada forzosamente por una nueva . "Mit dem Verschwinden der Ruinen, die in Grünflächen und Parkanlagen verwandelt oder durch Neubauten ersetzt wurden, verlor die Erinnerung an die unversehrte Stadt ihren letzen Halt [...]" (Maron 2003: 51-52).

En Zwischenspiel [Interludio] (2013) de nuevo un acontecimiento externo lleva a la narradora a revisar desde el presente su pasado. Ruth recibe la llamada de su hija Fanny para reunirse con ella en el funeral de Olga. En la novela existen numerosas referencias a obras anteriores. Como en Die Überläuferin, la protagonista considera el espacio de su memoria como si fuera un sótano, donde es capaz de recuperar y destruir los recuerdos a su antojo. "Er [Bernhard] lag in meinem Gedächtnis irgendwo archiviert, vergraben unter Späterem, das auch vergraben war unter noch Späterem, eine Erinnerungsleiche, farblos, geruchlos, kalt" (Maron 2013: 12). La figura en la novela del camarada Keller guarda relación a su vez con el personaje de Beerenbaum de Animal triste, cuyo infarto considera ahora merecido por su arrogante vida como Secretario del Partido.

A pesar de su resistencia a abandonar este espacio cerrado, seguro de memoria, Ruth toma el coche para acudir al cementerio. Sin embargo, nunca llega. "Ich nahm mir vor, sofort anzurufen, sobald ich zu Hause war, und ihr zu erzählen, wie es dazu gekommen war, dass eine kleine rückwärtsfliegende Wolke mich zuerst geblendet, in der Folge dann auf Umwege geführt hatte" (Maron 2013: 155). De nuevo el control sobre el ayer se resquebraja en el momento en que Ruth recorre el espacio de Berlín. En él se le aparece la propia difunta. Mientras que Ruth defiende la voluntad de reprimir el pasado, Olga aboga por la necesidad de recordar. El encuentro propicia la retrospección en la narración, en la que la figura vuelve una vez más a confrontarse con su biografía. "Eigentlich hatte ich nicht das Gefühl, dass es schon Zeit sei für ein Lebensresümee, aber an Tagen wie diesem drängten sich diese sinnlosen Fragen nach dem Woher und Warum [...]" (Maron 2013: 17). 
Por un lado, Maron viaja a través del tiempo de la ciudad mediante el trayecto al cementerio para escenificar otra vez la partición de Berlín. Un ejemplo es la referencia al antiguo túnel fronterizo de Wollankstraße. "Ich war froh als ich endlich die S-Bahn-Brücke an der Wollankstraße sah, die früher zur Mauer zwischen Ost- und Westberlin gehörte, wo der Wedding endete und Pankow begann" (Maron 2013: 39). Por otro lado, como en Geburtsort Berlin, la situación de espaciación del tiempo, que acontece al principio de la novela, se convierte, al igual que en el ensayo, en una nueva situación de temporalización del espacio. La salida de Ruth al exterior provoca la pérdida de su dominio del tiempo, lo que se refleja en la obra cuando afirma: "(d)ie winzigen Zeiger auf meiner Uhr konnte ich immer noch nicht erkennen" (Maron 2013: 152).

Este abandono del espacio protegido de la casa deja a la narradora a la intemperie de la ciudad, donde se abre una suerte de caja de pandora. En Geburtsort Berlin la ciudad se relaciona con un pasado fundamentalmente positivo. Sin embargo, en Zwischenspiel el espacio urbano fuerza a Ruth a revivir sus traumas de la infancia. "Ich habe Angst vor solchen Menschen, wahrscheinlich ein Relikt aus der Kindheit" (Maron 2013: 139). Entre las apariciones espectrales, que Ruth se imagina por las calles de Berlín, toman parte también Erich y Margot Honecker. A través de la persecución de dichas figuras, que la persiguen por el espacio urbano porfiando sus viejos discursos, Maron vuelve a entrelazar en la obra el destino personal con una historia nacional que acecha siempre latente.
Por último, Ruth logra paradójicamente recobrar su visión con el crepúsculo. La caída de la noche pone fin a un episodio que, pese a todo, tiene visos de no ser esporádico. Maron representa la ciudad como un espacio inseguro donde la memoria obedece a mecanismos involuntarios. Con ello la autora pretende poner de manifiesto la dificultad de proyectarse hacia el futuro en un entramado urbano que le sigue forzando con sus ecos en la dirección contraria. Como se reitera en el diálogo entre Ruth y Olga en la novela: "Solange Du in unserer Erinnerung lebst, bist Du nicht tot" (Maron 2013: 32).

\section{Conclusión}

En sus escritos Maron concede al espacio un papel destacado a fin de reconstruir el pasado desde el presente para valorarlo. Maron utiliza normalmente un espacio cerrado para escenificar el ayer como un estado temporal, al que tiene acceso sin restricciones y puede alterar motu proprio. Esta situación se rompe en el momento en que sale al exterior y debe adaptarse a los cambios históricos, sociales y políticos de su entorno. Frente a la espaciación del tiempo de sus primeras novelas, donde el espacio interviene como cómplice, en la temporalización del espacio, que se tiene lugar en obras más recientes, dicho espacio se convierte en un adversario del recuerdo. La dificultad de la pérdida por la inevitable adaptación o la involuntariedad de la memoria, que Maron escenifica durante sus paseos por la capital berlinesa, permite reflejar los problemas de la conciliación de lo vivido con el proceso de constitución, aun pasadas ya casi tres décadas, de la nueva identidad alemana.

conmemorativas, museos, archivos, bibliotecas, etc. Se hace evidente que en la mayoría de los casos se trata de espacios con los que el sujeto mantiene una especial relación de afecto.

5 Según Hinojosa, “(es) una constante en las novelas de Monika Maron que las protagonistas dediquen gran parte de su tiempo presente a rememorar experiencias pasadas, ya que en torno a ellas se construye la trama narrativa" (Hinojosa 2015: 155).

6 La novela está concebida sin embargo antes de la caída del Muro. Como destaca Sainz, la intuición de Maron al redactar la obra "es muy certera al advertir, a mediados de los años ochenta, una imparable crisis y decadencia sociopolítica de la RDA" (Sainz 2009: 223).

7 Según Hinojosa "(e)l acto de recordar concede a las protagonistas de Maron la oportunidad de otorgar coherencia interna a sus respectivas trayectorias vitales" (Hinojosa 2015: 155). El tratar de encajar los sucesos históricos le lleva a tener que poner en orden los suyos propios (Hinojosa 2015: 154).

8 Recientemente ha salido a la luz que Maron llegó a colaborar incluso durante unos años en la década de los setenta con el servicio estatal de espionaje. Según Sainz (2009: 221), "estas circunstancias la situaron en un lugar privilegiado entre los ciudadanos de su país". Maron obtuvo varios permisos para abandonar la RDA, hecho que se consumó en 1988 con su marcha a Hamburgo. 
9 Sobre la cuestión del proceso de reunificación Maron publica asimismo Zwei Brüder (2010). La escritora reconstruye en esta recopilación de 16 ensayos la historia del país comparándola con la relación entre dos hermanos. Según Hinojosa, las vidas de Helmut (o la República Federal de Alemania) y Manfred (o la República Democrática Alemana) siguen trayectorias distintas tras haber cometido un mismo delito (la guerra y el nacionalsocialismo). Mientras que Helmut se hace un empresario de éxito, Manfred ha de cumplir condena en la cárcel para expiar la culpa de los dos. Tras su puesta en la libertad, la complicidad que los une en el pasado se vuelve sospecha, lo que impide su nueva vida en común. "A la impaciencia de uno, que espera que el hermano se adapte rápidamente al nuevo ritmo de vida capitalista, y la falta de iniciativa del otro, que se repliega de esa sociedad refugiándose en su victimismo, achaca Maron el problema [...] que hace inviable la comunicación" (Hinojosa 2015: 160-161).

10 Según Hinojosa, “(a)nte la aparente dificultad de resolver las diferencias a través del diálogo [...], Maron empieza a contemplar la necesidad de olvidar para recordar, que es lo que Aleida Assmann denomina 'ein konstruktives Vergessen im Dienste eines hoffnungsvollen Neubeginns'”' (Hinojosa 2015: 162).

11 Se trata de una fórmula recurrente en las notas necrológicas de los periódicos, con la que Maron enfatiza la dificultad de olvidar.

Bibliographie

Obras:

Monika, M. (1981), Flugasche, Fischer, Frankfurt a. M.

Maron, M. (1986), Die Überläuferin, Fischer, Frankfurt a. M.

Maron, M. (1996), Animal triste, Fischer, Frankfurt a. M.

Maron, M. (1998), "Ich war ein antifaschistisches Kind", en Gibson, E. (ed.) (2006), Doch das Paradies ist verriegelt... Zum Werk von Monika Maron, Fischer Frankfurt a. M., 16-30.

Maron, M. (1999), Pawels Briefe. Eine Familiengeschichte, Fischer, Frankfurt a. M.

Maron, M. (2002), Endmoränen, Fischer, Frankfurt a. M.

Maron, M. (2003), Geburtsort Berlin, Fischer, Frankfurt a. M.

Maron, M. (2010), Zwei Brüder. Gedanken zur Einheit 1989-2009, Fischer, Frankfurt a. M.

Maron, M. (2013), Zwischenspiel, Fischer, Frankfurt a. M.

\section{Estudios:}

Assmann, J. (1992), Das kulturelle Gedächtnis. Schrift, Erinnerung und politische Identität in frühen Hochkulturen, München, C. H. Beck.

Assmann, A. (1999), Erinnerungsräume. Formen und Wandlungen des kulturellen Gedächtnisses, München, Beck.

Bachelard, G. (1957), La poétique de l'espace. Presses Universitaires de France, París.

Basseler, M. y Birke, D. (2005), "Mimesis des Erinnerns", en Erll, A. y Nünning, A. (eds.), Gedächtniskonzepte der Literaturwissenschaft.

Theoretische Grundlegung und Anwendungsperspektiven, Walter de Gruyter, Berlín [etc.], 123-147.

Boll, K. (2002), Erinnerung und Reflexion. Retrospektive Lebenskonstruktionen im Prosawerk Monika Marons, Könighausen \& Neumann, Würzburg.

Erll, A. (2005), "Literatur als Medium des kollektiven Gedächtnisses", en Erll, A. y Nünning, A. (eds.), Gedächtniskonzepte der Literaturwissenschaft. Theoretische Grundlegung und Anwendungsperspektiven, Walter de Gruyter, Berlín [etc.], 249-276.

Eschering, U. (1996), "Die Liebe, das andauernde Mysterium", en Das Borsenblatt 13/13, feb. 1996, 9-11.

Forte, D. (2005) “Über das Erzähhlen. Dankrede zur Verleihung des Niederrheinischen Literaturpreises der Stadt Krefeld 2005" en Hosemann, J. (ed.) (2007), »Es ist ein eigenes Schreiben ...« Materialien zum Werk von Dieter Forte, Frankfurt a. M., Fischer.

Hinojosa, O. (2010), Ficción histórica y realidad literaria. Análisis neohistoricista del Socialismo en la obra de Monika Maron, Peter Lang, Berna.

Hinojosa, O. (2015), El retorno al origen: adaptación y memoria en las crónicas de Mónica Maron en Revista de Filología Alemana, vol. 23, 153-168.

Humphrey, R. (2005), "Literarische Gattung und Gedächtnis”, en Erll, A. y
Nünning, A. (eds.), Gedächtniskonzepte der Literaturwissenschaft.

Theoretische Grundlegung und Anwendungsperspektiven, Walter de Gruyter, Berlín [etc.], 73-96.

Maldonado Alemán, M. (2010), "Verortung der Erinnerung. Raumdarstellung und Geschichtskonstruktion in Günter Grass ${ }^{\text {" }}$ Ein weites Feld" en Revista de Filología Alemana, vol. 18, 163-180.

Merck Navarro, B. (2009), "Historias de la unificación", en Maldonado Alemán, M. (coord.), Literatura e identidad cultural. Representaciones del pasado en la narrativa alemana a partir de 1945, Peter Lang, Berna, 367-401.

Pytel-Bartnik, E. (2010), Monika Marons Geburtsort Berlin (2003) - Die Stadt als Archiv der Erinnerung en Gansel, C. y Zimniak, P. (eds.), Das »Prinzip Erinnerung « in der deutschsprachigen Gegenwartsliteratur nach 1989, Vadenhoeck \& Ruprecht, Göttingen, 179-180.

Rossbacher, B. (1997), "The Status of State and Subject: Reading Monika Maron from Flugasche to Animal triste" en Weninger, R. y Rossbacher, B. (eds.), Wendezeiten, Zeitenwenden: Positionbestimmungen zur deutschsprachigen Literatur 1945-1995, Stauffenburg, Tübingen, 193-214.

Sainz Lerchundi,A. (2009), "Maron, Monika” en Maldonado Alemán, M. (coord.) (2009), La narrativa de la unificación alemana. Autores yobras, Peter Lang, Berna, 221-230. 